\title{
Emotional Processing of Harmonic Expectancy Violations
}

\author{
NIKOLAUS STEINBEIS, ${ }^{a, b}$ STEFAN KOELSCH, ${ }^{a}$ AND JOHN A. SLOBODA ${ }^{b}$ \\ ${ }^{a}$ Max-Planck-Institute for Human Cognitive and Brain Sciences, \\ 04103 Leipzig, Germany \\ ${ }^{b}$ University of Keele, Keele, United Kingdom
}

\begin{abstract}
This study is about whether harmonic expectancy violations can trigger emotional processes, as indexed by physiological and subjective measures.
\end{abstract}

KEYWORDS: harmonic expectancy; emotion; Bach chorales; EDA; EEG

\section{INTRODUCTION}

Music is capable of eliciting strong emotions in listeners. ${ }^{1}$ It has been argued that a reason for this is the suspension and fulfilment of implicit musical expectations of the listener and that music becomes increasingly emotional with the suspension of musical expectations. ${ }^{2}$ The validity of this claim was directly tested in this study.

It has been shown that listeners have harmonic expectations given a salient harmonic context. ${ }^{3}$ These expectations mirror the relationships of harmonic keys in Western music. Previous electroencephalogram (EEG) experiments have indicated event-related potentials (ERPs) to be sensitive to the harmonic context and its violation, as reflected by an early right anterior negativity (ERAN). ${ }^{4}$ In turn, it has been demonstrated that listeners reported heart racing when a prominent event occurred earlier than expected and shivers in response to a new or unprepared harmony. ${ }^{1}$ However, there is yet no direct evidence that the systematic manipulation of harmonies leads to an increase in emotions.

The purpose of the present study was to investigate the influence of three different degrees of harmonically expected harmonizations of the same pieces of music on the neural processing of these events, as well as on subjective and psychophysiological indicators of emotion.

Address for correspondence: Nikolaus Steinbeis, Max-Planck-Institute for Human Cognitive and Brain Sciences, Junior Research Group, Neurocognition of Music, Stephanstr. 1A, 04103 Leipzig, Germany. Voice: +49-341-355-217-10.

steinb@cbs.mpg.de

Ann. N.Y. Acad. Sci. 1060: 457-461 (2005). ㅇ 2005 New York Academy of Sciences.

doi: 10.1196/annals.1360.055 


\section{METHOD}

\section{Participants}

Twelve musicians (mean years of musical training: 13.8 years) and 12 nonmusicians participated in the study. Mean age was 25.15 years. Twelve of the participants were male.

\section{Stimuli}

Six Bach chorales were chosen explicitly for sections containing unexpected harmonies. This unexpectedness was measured using the circle of fifths, which demonstrates the distance between the 12 major and minor keys of Western music. Harmonically expected and very unexpected alternatives to a single cadential ending in each chorale were composed, by varying the distance of the target chord along the circle of fifths. Eventually there were three versions of each chorale, producing 18 stimuli in total.

\section{Materials}

\section{Emotional Self-Report}

This was the first part of the study, for which subjects heard chorales in their entirety. At the end of each chorale, participants stated how emotional they felt in response to the music.

\section{Electrodermal Activity}

Several studies have shown that emotions in response to music can be captured by psychophysiological measures. ${ }^{1,5}$ Electrodermal activity (EDA) has been suggested to be a good indicator of emotions, reflecting its arousal dimension, ${ }^{6}$ also while listening to music. ${ }^{7}$ Two electrodes were applied to the medial phalanx of the middle and index fingers of the nondominant hand.

\section{Electroencephalogram}

An EEG was recorded from 30 locations distributed over the scalp to study the neural processing of expectancy violations. EDA and EEG were recorded simultaneously for the second part of the study, in which shortened versions of the chorales were presented randomly.

\section{Procedure}

For the second part, participants had to perform a judgement task to show that they were paying attention to the music. The length of each stimulus had to be compared with the previously heard one and judged if it was shorter or longer. There was an 8-s pause between each response and the start of a new stimulus to allow the EDA to return to a baseline. 


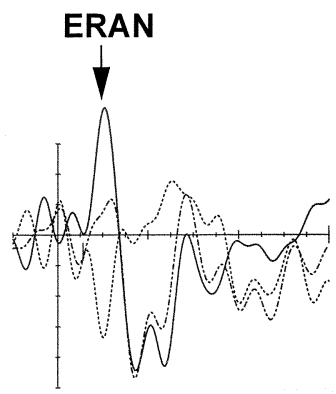

\section{Musicians}
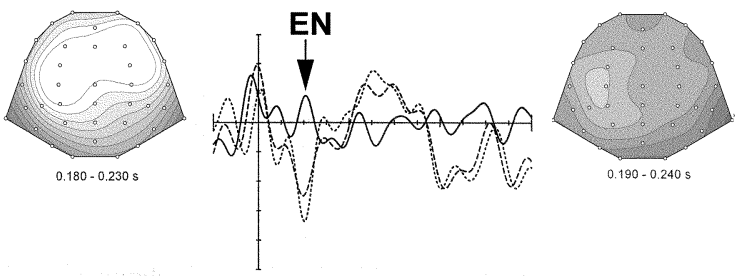

\section{Nonmusicians}
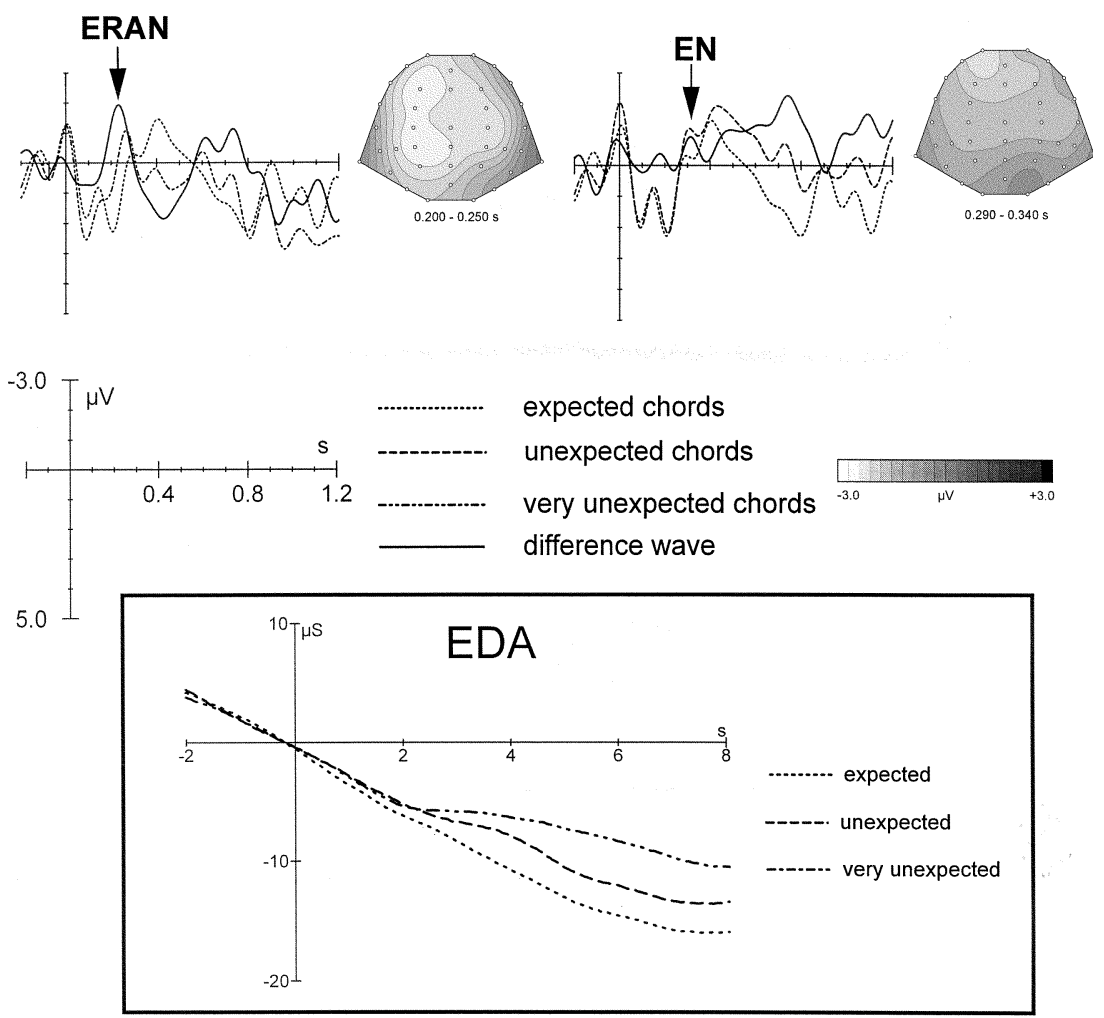

FIGURE 1. ERPs to harmonic expectancy violations. The difference waves indicate the processing of the violations (very unexpected-expected $=$ ERAN; unexpected-expected $=\mathrm{EN}$ ). The time course of the ERPs are shown from the F4 (right-anterior) electrode only, whereas adjacent scalp distributions display their strengths over all electrodes. Because of group differences in peak latency, the data for musicians and nonmusicians are displayed separately. The EDA data (bottom) are shown for all subjects. 


\section{RESULTS}

\section{Event-Related Potentials}

The difference between ERP responses to the very unexpected and the expected harmonic events displays an ERAN for both groups (statistic from frontal electrodes: $F\{1,22\}=11.43 ; P<.005)$, which occurred slightly earlier for musicians than for nonmusicians $(t\{22\}=2,048 ; P=.053)$.

The difference between responses to the unexpected and the expected harmonic events also reveals a negativity for both groups $(F\{1,22\}=4.99 ; P<.05)$, which occurred considerably earlier for musicians than nonmusicians $(t\{22\}=-3.585$; $P<.005)$. Because it is neither lateralized to the right, nor stronger over anterior leads, this negativity will be referred to as an early negativity (EN). It is assumed to reflect the same neural processes as the ERAN. This view is supported by a marginally significant difference in negativity size $(F\{1,22\}=3.99 ; P=.058)$, suggesting a sensitivity to increasing harmonic unexpectedness.

\section{EDA and Subjective Response}

The EDA increased in response to harmonic unexpectedness over time $(F\{2,46\}=6.15 ; P=.01)$. Equally, listeners reported an increased emotionality at the end of chorales containing increased harmonic unexpectedness $(F\{2,141\}=$ $17.591 ; P=.0001)$. Because this increase was systematic with harmonic unexpectedness, this suggests that heightened harmonic unexpectedness is increasingly emotional (FIG. 1).

\section{CONCLUSION}

The data suggest that harmonically unexpected events do elicit emotional effects. Whereas the EEG data demonstrate that early negativities are sensitive to the degree of harmonic expectancy violation, the subjective and physiological data suggest that these violations predispose subjects to an increased emotionality. It can be argued that the violations lead to an increase in physiological "arousal," which in turn leads to a heightened emotional experience of the musical pieces. These data cannot be explained by manipulated versus original Bach, because the unexpected harmonies are the original compositions.

The role of the early negativities remains to be specified. It seems most likely that the detection of a harmonic expectancy violation will trigger a variety of different processes, including emotional ones. Recent neuroimaging work reports the activation of areas involved in emotional processing, such as the orbital frontolateral cortex, in response to harmonic expectancy violations. ${ }^{8}$ In conjunction with the present data, this provides compelling support for the idea that suspensions of musical expectations are an important pathway to generating emotions in the listener. 


\section{ACKNOWLEDGMENT}

This research was kindly supported by an award from the Arts and Humanities Research Board to Nikolaus Steinbeis.

[Competing interests: The authors declare that they have no competing financial interests.]

\section{REFERENCES}

1. Sloboda, J.A. 1991. Music structure and emotional response: some empirical findings. Psychol. Mus. 19: 110-120.

2. MeYer, L.B. 1956. Emotion and Meaning in Music. University of Chicago Press. Chicago.

3. Bharucha, J.J. \& K. Stoeckig. 1986. Reaction time and musical expectancy: priming of chords. J. Exp. Psychol. Hum. Percept. Perform. 12: 403-410.

4. Koelsch, S., T. Gunter, A.D. Friederici, et al. 2000. Brain indices of musical processing: "nonmusicians" are musical. J. Cogn. Neurosci. 12: 520-541.

5. KRumhansl, C.L. 1997. An exploratory study of musical emotions and psychophysiology. Can. J. Exp. Psychol. 51: 336-352.

6. Bradley, M.M. \& P.J. Lang. 2000. Affective reaction to acoustic stimuli. Psychophysiology 37: 204-215.

7. Khalfa, S., I. Peretz, J. Blondin, et al. 2002. Event-related skin conductance responses to musical emotions in humans. Neurosci. Lett. 328: 145-149.

8. Koelsch, S., T. Fritz, K. Schulze, et al. 2005. Adults and children processing music. An fMRI study. NeuroImage 25: 1068-1076. 